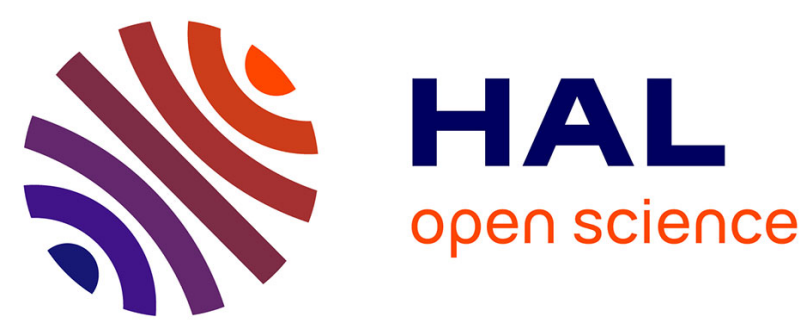

\title{
Tomographic reconstruction of tokamak edge turbulence from single visible camera data and automatic turbulence structure tracking
}

Jordan Cavalier, Nicolas Lemoine, Frédéric Brochard, Vladimir Weinzettl, Jakub Seidl, Scott Silburn, Patrick Tamain, Renaud Dejarnac, Jiri Adamek, Radomir Panek

\section{To cite this version:}

Jordan Cavalier, Nicolas Lemoine, Frédéric Brochard, Vladimir Weinzettl, Jakub Seidl, et al.. Tomographic reconstruction of tokamak edge turbulence from single visible camera data and automatic turbulence structure tracking. Nuclear Fusion, 2019, 59 (5), pp.056025. 10.1088/1741-4326/ab0d4c . hal-02106648

\section{HAL Id: hal-02106648 \\ https://hal.science/hal-02106648}

Submitted on 20 Nov 2019

HAL is a multi-disciplinary open access archive for the deposit and dissemination of scientific research documents, whether they are published or not. The documents may come from teaching and research institutions in France or abroad, or from public or private research centers.
L'archive ouverte pluridisciplinaire $\mathbf{H A L}$, est destinée au dépôt et à la diffusion de documents scientifiques de niveau recherche, publiés ou non, émanant des établissements d'enseignement et de recherche français ou étrangers, des laboratoires publics ou privés. 


\title{
Tomographic reconstruction of tokamak edge turbulence from single visible camera data and automatic turbulence structure tracking
}

\author{
Jordan Cavalier ${ }^{1,2}$, Nicolas Lemoine ${ }^{2}$, Frederic Brochard ${ }^{2}$, Vladimir Weinzettl ${ }^{1}$, Jakub Seidl ${ }^{1}$, \\ Scott Silburn ${ }^{3}$, Patrick Tamain ${ }^{4}$, Renaud Dejarnac ${ }^{1}$, Jiri Adamek ${ }^{1}$, and Radomir Panek ${ }^{1}$ \\ ${ }^{1}$ Institute of Plasma Physics of the CAS, Prague, Czech Republic \\ ${ }^{2}$ Institut Jean Lamour IJL, Université de Lorraine, Vandouvre-lès-Nancy, France \\ ${ }^{3}$ CCFE, Culham Science Centre, Abingdon, Oxon, OX14 3DB, United Kingdom \\ ${ }^{4}$ CEA, IRFM, F-13108 Saint-Paul-lèz-Durance, France
}

February 18, 2019

\begin{abstract}
Following the lead of [Nguyen van Yen Nucl. Fus. 52 (2012) 013005 (11pp)], this article tackles the problem of tomographic inversion with one camera assuming a constant emissivity of light along the magnetic field lines. In this way, the $3 \mathrm{D}$ problem reduces to $2 \mathrm{D}$ by helical symmetry, allowing for the reconstruction of any poloidal plane in the field of view of the camera. It is shown in this article that the complexity of using a wavelet basis for the reconstruction, as presented by Nguyen van Yen, is not necessary. The method is also validated by confronting it to 3D numerical data coming from the TOKAM3X code, for which the emissivity is slowly varying along the field lines (up to $20 \%$ ), showing the robustness of the reconstruction. The technique is then applied to real camera data recorded during a D-shaped ohmic plasma shot realized in the COMPASS tokamak. The method is experimentally validated by comparing reconstructed data from camera and ion saturation currents measured by Langmuir probes in the divertor region. Finally, it is shown that automatic detection and tracking of structures visible in the reconstructed poloidal plane enables unique investigations of edge plasma physics and opens wide perspectives for this method.
\end{abstract}

\section{Introduction}

Edge plasma turbulence in toroidal magnetic fusion devices plays a significant role on particle and energy confinement as well as on plasma-wall interactions $[1,2,3]$. Several diagnostics can be used to study this region, which extends radially from the material wall of the device to the vicinity of the separatrix, like electrotatic probes, beam emission spectroscopy, visible imaging, reflectometry... However, the interpretation of turbulence measurements remains difficult. In particular, visible imaging measures light emission resulting from the interaction between the plasma and neutral particles. In most tokamaks and stellarators, turbulence imaging is based on the local injection of neutral gas to enhance visible light emission. This technique, called gas puff imaging (GPI), facilitates the visualization of turbulent structures by increasing the signal-to-noise ratio and by localizing measurements to a nearly 2D cross-section $[4,5,6,7]$. GPI enables excellent space-time resolution of fluctuations, making it possible to investigate scales ranging from $0.1 \mathrm{~mm}$ to a few centimeters and from a few $\mathrm{kHz}$ to about $1 \mathrm{MHz}$, thus covering the essential parts of the fluctuation spectrum in the concerned region. However, GPI is not passive and causes perturbations to the plasma. These perturbations can be evidenced by other diagnostics, but their dependence on many parameters makes them rather difficult to evaluate $[4,8,9]$.

In this paper, we focus on the use of passive turbulence imaging, i.e. without any gas puff. This technique is intrinsically non-perturbative, but it suffers mostly from two disadvantages. First, the collected visible light can be very weak compared to GPI, resulting in a lower signal-to-noise ratio. Second, measurements are not localized due to the line-of-sight (LOS) integration over the emission sources. Low visible light conditions are still an issue, which is however getting less and less critical as modern fast cameras get more and more sensitive. It is also possible to handle the issue of line-of-sight integration by performing tomographic inversion, as it was done with various approaches on several toroidal devices $[10,11,12,13]$. With appropriate processing, it is possible to extract useful information from plasma visible light emission. We present here tomographic inversion applied to experimental video data recorded on the COMPASS tokamak [14] without any help of gas puff. The method is first illustrated 
with an academic case and validated against simulation data of the 3D fluid turbulence code TOKAM3X. Then, comparisons with probe measurements demonstrate its capability to reconstruct high-frequency fluctuations in any $2 \mathrm{D}$ plane intersecting the viewing volume. As we finally show, this makes it possible to analyze reconstructed data statistically without any temporal averaging, providing high-resolution information of structure's dynamics.

\section{Scrape-of-layer observation by fast visible camera on COMPASS}

The scrape-of-layer (SOL) of the COMPASS tokamak was observed with a SA-X2 photron camera at a frame rate of $270 \mathrm{kfps}$ and an exposure time of about $2.1 \mu \mathrm{s}$. At this speed, the resolution of the CMOS sensor is $128 \times 144$ pixels, with each pixels having a 20x20 $\mu \mathrm{m}^{2}$ surface. Thanks to its high sensitivity (25 $000 \mathrm{ISO}$ ) and dynamic range (12-bits), the light fluctuation of the plasma edge was observed with no need for local gas injection (GPI), i.e. the natural interaction between the neutral gas naturally present in the SOL and the plasma was enough to study the edge turbulence. The visible spectral range of the camera lies in $[400 ; 1000] \mathbf{n m}$. During the COMPASS discharge we are studying in this article, spectrometer measurements shows that radiations are mainly coming from the $D_{\alpha}$ line $(656 \mathrm{~nm})$, for which the sensitivity of the camera is almost maximum.

The camera was placed at the angular lower port (below the midplane) located in the $4 / 5$ section of the device (see Fig. 2.1.a), integrated to the rapid imaging system of COMPASS [15] and an aluminium mirror of $21 \mathrm{x} 44$ $\mathrm{mm}^{2}$ was installed in-vessel to collect the light coming from a zone centered around the last closed flux surface for D-shaped plasmas (see Fig. 2.1.b and c). In addition, the mirror was tilted in such a way that the line-of-sights (LOS) were locally tangential to the magnetic field lines at a distance of about $30 \mathrm{~cm}$ from the mirror location for the considered discharges. Therefore, a $16 \mathrm{~mm}$ focal length objective was used with the camera and focused at about $30 \mathrm{~cm}$. Because the camera is sensitive to the magnetic field generated by the coils surrounding the vessel, a telescope-like setup was used to put the camera as far as possible from the stray magnetic field. This setup consists of two identical objectives of $75 \mathrm{~mm}$ focal length placed in opposite directions and focused at infinity so that the rays in between the two objectives are parallel to the optical axis providing that the rays comes from the focal plane of the first objective. Consequently, the $16 \mathrm{~mm}$ objective creates an image in the focal plane of the first objective and the camera is located in the one of the second objective. Therefore, the camera could be located at about $1 \mathrm{~m}$ from the vessel thanks to black extension tubes in between the two $75 \mathrm{~mm}$ optics (see Fig. 2.1.a for a scheme of the optical apparatus). This optical configuration allows a resolution of about $0.4 \mathrm{~mm}$ between two consecutive pixels at $28 \mathrm{~cm}$ from the mirror and about $0.7-0.8 \mathrm{~mm}$ at $65 \mathrm{~cm}$ (i.e. at the vessel wall and slightly unfocused).

Performing tomographic reconstruction requires a precise localization of the camera and determination of the field of view in the lab frame. On COMPASS, this calibration is done using the Calcam software [16] that compares an image of the real field of view taken with the camera while enlightening the inside of the vessel and the CAD representation of the machine. In this particular case, the field of view being very narrow and since very few features on the vessel were visible, the CAD model was adjusted manually to fit as much as possible the calibration picture (see Fig. 2.1.d).

As an illustration of how data look like, the left column of Fig. 2.2 shows three successive snapshots taken in the D-shape plasma discharge \#15487 during the flattop phrase (discharge time 1150 ms). Since the camera has a 12-bit dynamic, only the highest bits have been considered to display the images. One can notice that structures are hardly distinguishable when looking at the raw data. In addition, stripes can be seen in both horizontal and vertical directions. They are present there due to the different sensitivities of each sensor composing the camera chip that was not corrected before recording the video. To reveal hidden structures, a sliding median image calculated over 10 frames around the considered image has been subtracted from the raw data and is shown in the central column of Fig. 2.2. Filamentary-like structures are then revealed, even though a non negligible noise is present. The structures above the sliding median will be refered as positive structures while the one smaller than the median will be refered as negative. Note that the stripes due to the sensor sensitivity disappeared and did not affect sensibly the fluctuating part of the signal. However, a strong shot noise is present, estimated to affect the signal by about $35 \%$. To further process the signal, a spatial Gaussian filter with a standard deviation of 5 pixel was applied, smearing out structures smaller than $\sim 2 \mathrm{~mm}$. The noise is smeared out, the essential part of the signal is retrieved but the filament dynamic stays complex for the human eye. Also note that the camera images are presented according to the experimental setup orientation, i.e. low-field side (LFS) to the left, while we present later on poloidal cuts with the opposite convention to be consistent to what is usually shown in the fusion community. 
a)

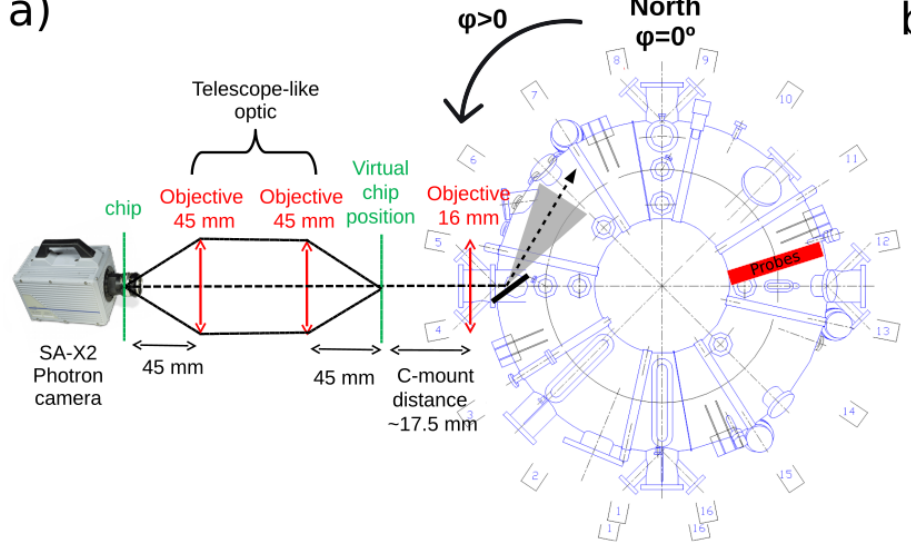

b)

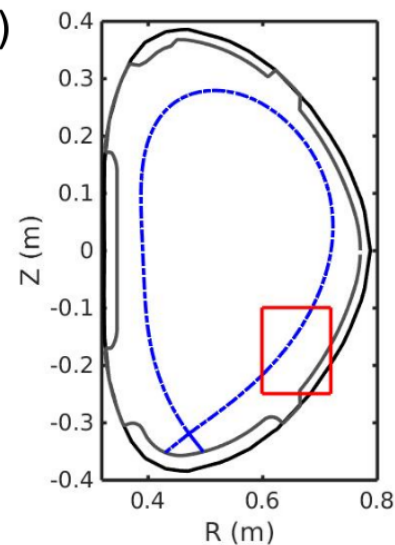

c)

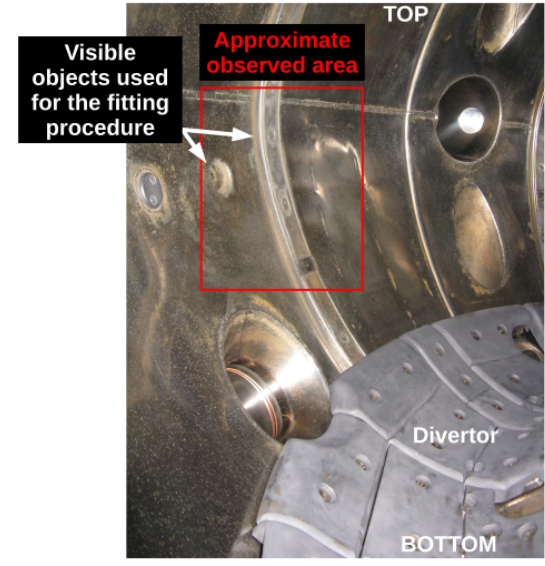

d)

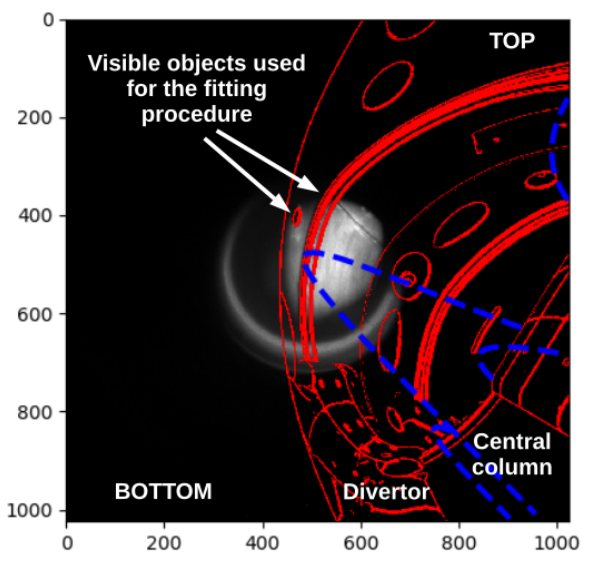

Figure 2.1: a) Schematic of the optical apparatus installed on COMPASS. The location of the probe array that will be used in Section 4.3 is also shown, as well as the convention taken for the angle $\varphi$. b) Approximated field of view of the camera in a poloidal cross-section in the focal plane of the optical apparatus. Note that the cross-section is mirrored compared to the real observation to keep usual COMPASS conventions. c) In-vessel picture taken with a standard camera during vessel opening. d) Rendering of the Calcam calibration on top of a full-frame calibration picture taken with the SA-X2 camera while illuminating the open vessel. The blue line represents a typical fieldline passing in the field of view of the camera. 

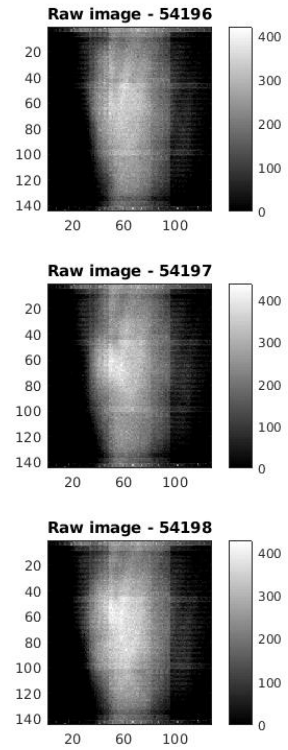
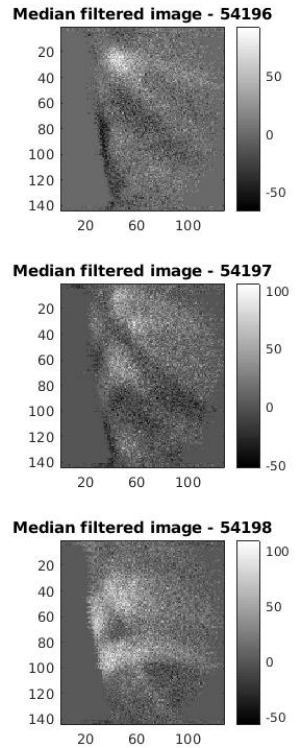

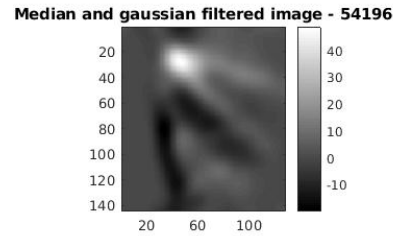

Median and gaussian filtered image - 54197

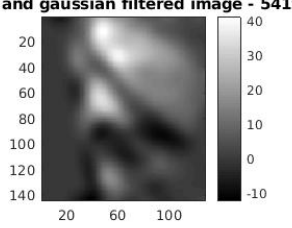

Median and gaussian filtered image - 54198

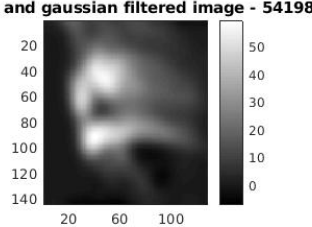

Figure 2.2: Left: three successive snapshots of the D shaped discharge \#15487 taken at a sampling frequency of $270 \mathrm{kfps}$ and an exposure time of $2.1 \mu \mathrm{s}$. Middle: Images obtained after subtraction of a median image calculated over 10 frames around the considered picture. Right: Medianed filtered images after applying a spatial Gaussian filter with a standard deviation of 5 pixel.

\section{Tomographic reconstruction}

Unlike gas-puff imaging experiments, the measurement presented in Fig. 2.2 is not located in a poloidal plane but is the result of the integration of the light along each line-of-sight of the camera, i.e. the image is the projection of a 3D structure onto the 2D camera chip. Therefore, information about the radial velocity or the perpendicular blob dimension is not straightforwardly infered. Years ago, a tomographic method has been proposed by Nguyen van Yen et al. [13] to recover the poloidal plane corresponding to circular edge plasma from camera data assuming constant light emissivity of the turbulent structures along the magnetic field lines. Other techniques exist to perform tomographic inversion in this case, such as the least-squares method or the singular value decomposition (SVD) reconstruction, but it has been shown that they are both less efficient than the technique used in Ref. [13]. To our knowledge, the method has not been used since then probably due to its rather complicated mathematical development and the high signal to noise ratio that the method requires to observe and follow fast movements of structures without any additional light source than neutral atoms naturally present in the SOL of the tokamak. However, cameras' sensitivity as well as their speed have improved significantly in the past years making the method relevant to fusion plasma edge turbulence studies. Due to the complexity of the method presented in Ref. [13], we recall in some details in this section the procedure that was used to recover fluctuation's strutures in a $2 \mathrm{D}$ poloidal cross-section from a 3D structure projected along the different lines of sight of the camera, assuming constant emissivity along the magnetic field lines. We also show that the projection basis does not necessary have to be a wavelet basis, simplifying the implementation of the method.

\subsection{General principle}

The camera apparatus is approximated by a screen collecting the light passing through a pinhole located at the pupil of the camera objective. Let $S_{0}(\psi, \theta, \varphi)$ be the plasma emissivity at a point $M$ characterized by its fieldline coordinates $(\psi, \theta, \varphi)$, where $\psi$ is a flux coordinate, $\theta$ a poloidal coordinate and $\varphi$ the toroidal angle. Assuming that radiations are isotropic, that the plasma is transparent and that radiations are mainly coming from $H_{\alpha}$ and $D_{\alpha}$ radiations so that the spectral response of the camera does not play a role, the intensity collected by the camera at the pixel $(x, y)$ is given by 


$$
I_{0}(x, y)=\int_{s_{c}}^{+\infty} S_{0}\left(\psi\left(s_{x y}\right), \theta\left(s_{x y}\right), \varphi\left(s_{x y}\right)\right) d s_{x y}
$$

where $(x, y)$ are the horizontal and vertical coordinates in the image plane, $s_{x y}$ is the curvilinear abscissa along the ray passing through $(x, y)$ and $s_{c}$ the position of the camera pupil.

The goal of the tomographic inversion is to retrieve the local plasma emissivity $S_{0}$ knowing the camera picture $I_{0}$ by inverting Eq. 3.1. The structures that one is willing to recover being three dimensional and the image 2D, it is therefore necessary to make one more assumption for the problem to be solvable. We thus assume that the emissivity of the observed structures is constant, or slowly varying, along the magnetic fieldlines within the field of view of the camera. This hypothesis is justified by the fact that the parallel velocity of the particles along the fieldlines is high [17] and, in the particular case of this article, that the view is narrow (about $\Delta \varphi=75^{\circ}$ is visible by the camera). Thanks to the helical symmetry of the fieldlines, the problem now reduces to recover the emissivity in any $2 \mathrm{D}$ poloidal cross section $S_{0}\left(\psi, \theta, \varphi_{r}\right)$, located at the toroidal angle $\varphi_{r}$, knowing the 2D image $I_{0}$.

\subsection{Transfer matrix creation}

This poloidal cross-section located at the toroidal angle $\varphi_{r}$ will be called in the following the reference poloidal plane. It is in that plane that the emissivity pattern will be reconstructed from the camera image. In order to handle the inversion numerically, the reference poloidal plane is discretized on a mesh grid, the image plane being already discretized by the pixels of the CCD sensor of the camera. Thus, any cell of the meshgrid of the reference poloidal plane $\left(\varphi=\varphi_{r}\right)$ is associated to a homogeneous structure inside the magnetic flux tube passing through this cell.

Let us now consider the mapping $\mathcal{K}$ that associates any emissivity pattern that can be built on the reference plane mesh grid to the image on the camera of the related 3D emission structure, given by helical symmetry along magnetic flux tubes. From Eq. 3.1, we know that $\mathcal{K}$ is a linear mapping. Thanks to the discretization of the reference poloidal plane, both the source set (the set of emissivity patterns in the reference plane) and the target set (the set of all the possible images on the CCD camera) are of finite dimension. Thus Eq. 3.1 can be written in terms of matrices, i.e., following the notation of Ref. [13], $I_{0}=K S_{0}$, where $S_{0}$ is the vector containing the emissivity of each cell of the mesh grid of the reference poloidal plane, $I_{0}$ the vector containing the intensity of each pixel of the CCD camera and $K$ is the matrix associated to the mapping $\mathcal{K}$.

Practically, we computed the transfer matix $K$ as follows:

- In order to avoid unnecessary calculation, we take into account the actual field of view of the camera. In a poloidal plane, a point is characterized by $R$, its distance to the main axis of the torus and $z$, its height, whose origin is the equatorial plane. We consider the path through the torus of the four extreme LOS passing by the camera pupil and by one of the four pixel corners of the CCD sensor of the camera. We compute the minimum radius $R_{\text {min }}$ reached by the most inner LOS, the maximum radius $R_{\text {max }}$ is taken as the radius of the external wall in the equatorial plane, the minimum and the maximum height $z_{\min }$ and $z_{\max }$ are the minimum and the maximum height of these four LOS inside the torus.

- The reference poloidal plane is then chosen within the field of view of the camera. We choose the reference plane to correspond to the plane where most of the LOS of the camera are tangential to the magnetic fieldlines as most of the useful signal will come from that plane and as it makes the tomographic inversion more robust to magnetic reconstruction uncertainties (see Section 4.3).

- The reference poloidal plane is discretized on a cartesian mesh grid. Any mesh cell center $G$ has coordinates $\left(R_{G}, Z_{G}\right)$ such that $R_{\min }<R_{G}<R_{\max }$ and $z_{\min }<Z_{G}<z_{\max }$.

- A look-up table is created, by integrating the magnetic lines passing by each mesh grid point using the magnetic topology calculated by the EFIT reconstruction software [18]. This makes it possible to associate any point inside the torus to the cell of the mesh grid of the reference poloidal plane that is magnetically connected to it (unless the point is not connected to any cell of the mesh grid).

- Numerical integration of Eq. 3.1 along each LOS to get the transfer matrix $K$. One LOS corresponds to one pixel on the CDD sensor whose coordinates in the sensor plane are $(x, y), x$ and $y$ being integer indexes. Let $M$ be a point on the LOS associated to the pixel $(x, y)$, at the center of an integration step. Let $\Delta l$ be the length of the integration step of the numerical integration at point $M$. If $M$ is magnetically connected to 
the cell $(i, j)$ of the mesh grid of the reference poloidal plane, then $K$ is incremented at the integration step centered on $M$ as follows

$$
K(x, y, i, j)=K(x, y, i, j)+\Delta l
$$

As a result, for one given cell of indexes $\left(i_{0}, j_{0}\right), K\left(x, y, i_{0}, j_{0}\right)$ is the image on the camera of the structure along the magnetic flux tube passing through this cell of index $\left(i_{0}, j_{0}\right)$, with an emissivity equal to one inside that magnetic flux tube and vanishing elsewhere (see Fig. $3.1 \mathrm{a}$ ) and b) for a practical example).

\subsection{Matrix inversion}

The method to calculate the elements of the transfer matrix $K$ being presented, we are now discussing and explaining the method to invert it. Explanations are admittedly already available in Ref. [13], but we think it is worth giving some more details for a better understanding. Discussion is mainly about the inversibility of the mapping $\mathcal{K}$. What are the conditions to fulfill for $\mathcal{K}$ to be inversible? In Ref. [13], inversibility is merely assumed and it was only observed numerically that the matrix $K$ was actually inversible in the case treated.

The strength of the method used in Ref. [13] is the way the matrix $K$ is built, as explained in the previous subsection. The transfer matrix $K$ yields the image on the camera screen of each magnetic flux tube whose crosssection in the reference poloidal plane is one cell of the cartesian mesh $(i, j)$. The ensemble of the images of those structures and all the linear combinaisons of them is a subset of the ensemble of the images that can be drawn on the camera.

A first necessary condition for the mapping $\mathcal{K}$ to be a one-to-one mapping (bijective), and thus inversible, is that this subset of the ensemble of the images is equal to the set of all images likely to be recorded during one shot by the camera as they are after the preprocessing described in Section 2. This implies that there is no structure smaller than the scale of the mesh grid and yet resolved by the camera. Notice that the gaussian filter that is applied to camera images, as explained in Section 2, not only removes noise but also removes or enlarges, if its width is properly choosen, too small structures, that cannot be reproduced by a linear combination of the images of the mesh magnetic flux tubes (i.e. the magnetic flux tubes passing through the different mesh cells). A great care should also be taken regarding the areas of the CCD sensor that collect no light due to obstacles between the plasma and the camera. They have to be discarded from the image grid.

Another necessary condition is to discard from the source set basis all the mesh cells in the reference poloidal plane whose corresponding flux tubes are invisible by the camera.

The last necessary condition is that the size of the cells of the mesh grid of the reference plane is large enough so that their images can be resolved by the camera (i.e. two different cells yield two distinguable images).

All those necessary conditions taken together seem to us sufficient to ensure that the mapping $\mathcal{K}$ is a one to one mapping. Then it is worth inverting the transfer matrix $K$, as it will make possible to get the shape of the structure cross-section in the reference poloidal plane, at the resolution of the chosen mesh grid. Consequently, the inversion method is now explained.

Let $\left(\psi_{\lambda}\right)_{\lambda \in\left[1 . . N_{\lambda}\right]}$ be an orthogonal basis of the reference poloidal plane mesh, where $N_{\lambda}$ is the number of nodes on the mesh in the reference poloidal plane whose image on the camera is non-zero. In such a basis, the light emissivity $S_{0}$ can be written as

$$
S_{0}=\sum_{\lambda=1}^{N_{\lambda}} s_{\lambda} \psi_{\lambda}=\sum_{\lambda=1}^{N_{\lambda}}\left\langle\psi_{\lambda} \mid S_{0}\right\rangle \psi_{\lambda}
$$

where $\langle. \mid$.$\rangle means dot product, both in the reference poloidal plane mesh grid and in the image plane. The image$ $I_{0}$ of $S_{0}$ by $\mathcal{K}$ reads

$$
I_{0}=K S_{0}=\sum_{\lambda=1}^{N_{\lambda}} s_{\lambda} K \psi_{\lambda}
$$

where $K \psi_{\lambda}$ is the matrix containing the intensity of each pixel related to the image on the camera of the basis vector of the reference poloidal plane $\psi_{\lambda}$. As discussed in the previous paragraph, the mesh grid in the reference poloidal plane and the Gaussian filter are chosen so that $\left(K \psi_{\lambda}\right)_{\lambda \in\left[1 . . N_{\lambda}\right]}$ is a basis of the ensemble of all the possible images that can be obtained from the camera after the preprocessing detailed in Section 2.

Then, it comes from Eq. 3.4 that if the decomposition of the preprocessed image on the basis $\left(K \psi_{\lambda}\right)_{\lambda \in\left[1 . . N_{\lambda}\right]}$ is found (i.e. the coefficients $s_{\lambda}$ ), the related emissivity field in the reference plane is recovered. The only difficulty is 
that the image by $K$ of the basis $\left(\psi_{\lambda}\right)_{\lambda \in\left[1 . . N_{\lambda}\right]}$ is not orthogonal. Thus to get the coefficients $s_{\lambda}$, the adjoint basis of $\left(K \psi_{\lambda}\right)_{\lambda \in\left[1 . . N_{\lambda}\right]}$ have to be processed.

Let $\mathcal{P}$ be the set of the structures on the reference poloidal mesh grid, reduced to the nodes whose image on the camera is non vanishing, and let be $\mathcal{I}$ the ensemble of the images on the areas of the CCD sensor illuminated by the plasma. Then, the adjoint basis is related to the adjoint operator $K^{*}$, defined as follows

$$
\forall(a, b) \in \mathcal{P} \times \mathcal{I},\langle K a \mid b\rangle=\left\langle a \mid K^{*} b\right\rangle
$$

Note that $K$ is defined on $\mathcal{P}$ whereas $K^{*}$ is defined on $\mathcal{I}$.

Now considering the $N_{\lambda}$ vectors of the orthonormal basis of $\mathcal{P}$ called $\left(\psi_{\lambda}\right)_{\lambda \in\left[1 . . N_{\lambda}\right]}$, as $K$ is inversible (and thus $\left.K^{*}\right)$, there exist two corresponding image families $\left(\chi_{\lambda}\right)_{\lambda \in\left[1 \ldots N_{\lambda}\right]}$ and $\left(\xi_{\lambda}\right)_{\lambda \in\left[1 \ldots N_{\lambda}\right]}$, both in the image plane $\mathcal{I}$ defined by

$$
\begin{aligned}
K \psi_{\lambda} & =\kappa_{\lambda} \chi_{\lambda} \\
K^{*} \xi_{\lambda} & =\kappa_{\lambda} \psi_{\lambda}
\end{aligned}
$$

where $\kappa_{\lambda}$ is chosen in order to impose $\left\|\xi_{\lambda}\right\|=1$ for all $\lambda$ (useful condition to apply the thresholding method described in the next section) and where $\|$.$\| is the norm in both \mathcal{P}$ and $\mathcal{I}$. The matrix $K$ being inversible, those two families are two basis of the set $\mathcal{I}$ and $\left(\xi_{\lambda}\right)_{\lambda \in\left[1 \ldots N_{\lambda}\right]}$ corresponds to the adjoint basis that we are looking for. The equation that gives the reconstructed emissivity in the reference poloidal plane $S_{0}$ can now easily be found from Eqs. 3.3, 3.5 and 3.6 and reads

$$
S_{0}=\sum_{\lambda=1}^{N_{\lambda}}\left\langle I_{0} \mid \xi_{\lambda}\right\rangle \kappa_{\lambda}^{-1} \psi_{\lambda}
$$

From Eq. 3.3 and 3.7, it comes that the coefficients of the $\psi_{\lambda}$ of the stucture in the reference poloidal plane read

$$
s_{\lambda}=\left\langle I_{0} \mid \xi_{\lambda}\right\rangle \kappa_{\lambda}^{-1}
$$

In the following, we call $s_{\xi_{\lambda}}$ the dot product of the image on the camera by the adjoint basis vector $\xi_{\lambda}$

$$
s_{\xi_{\lambda}}=\left\langle I_{0} \mid \xi_{\lambda}\right\rangle
$$

The last step that needs to be done is to find the $N_{\lambda}$ coordinates of the elements of the $\xi_{\lambda}$ basis, which is done by solving a $N_{\lambda}$ number of $N_{\lambda} \times N_{\lambda}$ linear systems obtained using the biorthogonality condition that results from Eqs. 3.5 and 3.6, i.e.

$$
\left\langle\xi_{\lambda} \mid \chi_{\lambda^{\prime}}\right\rangle=\left\{\begin{array}{cc}
1 & \text { if } \lambda=\lambda^{\prime} \\
0 & \text { otherwise }
\end{array}\right.
$$

\subsection{Basis choice and denoising}

To apply the method described above, it is then necessary to choose an orthogonal basis $\left(\psi_{\lambda}\right)_{\lambda \in\left[1 . . N_{\lambda}\right]}$ of the discrete reference poloidal plane. From the above demonstration, it is clear that the method does not require the basis of the discretized reference poloidal plane to be of any particular form for the inversion method to be correct. It just has to be orthogonal. For simplicity, we have chosen base vectors that correspond to each grid nodes of the reference poloidal plane that yields a non vanishing image on the camera CCD sensor with an emissivity equals to one, as they define an orthonormal base. An example of a $\psi_{\lambda}$ vector is represented in Fig. 3.1 as well as the $\chi_{\lambda}$ and $\xi_{\lambda}$ families obtained from Eq. 3.6.

Note that in Ref. [13] the method was applied to a basis of wavelet vectors, used mainly for denoising purpose (wavelet-vaguelette decomposition). Regarding the additional complexity brought by the use of wavelet theory, we have chosen a simpler basis and we will show in the next sections that the final results are good enough so that the use of wavelets, even for denoising, is not a necessary condition in our case. We used the same denoising technique as the one proposed in Ref. [13] that eliminates all coefficients $s_{\xi_{\lambda}}=\left\langle I_{0} \mid \xi_{\lambda}\right\rangle$ in Eq. 3.7 below a threshold $\Theta$ calculated from the data themselves. It is for this thresholding technique that the dual basis has to be normalized, otherwise the coefficients associated with the $\xi_{\lambda}$ with the smallest norms would be artificially large. The threshold is the limit of a sequence whose initial term is

$$
\Theta_{0}=\frac{c^{2}}{N_{\lambda}} \sum_{\lambda=1}^{N_{\lambda}} s_{\xi_{\lambda}}^{2}
$$



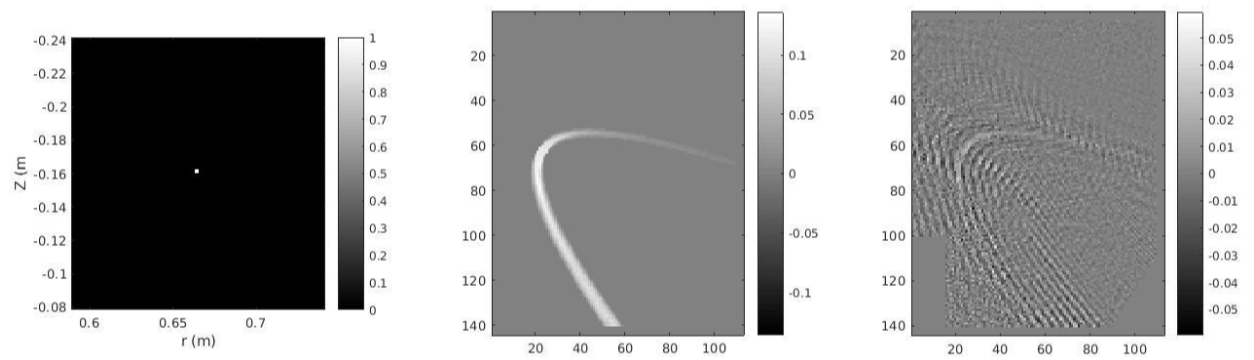

Figure 3.1: One example of a base vector $\psi_{\lambda}$ (a single grid node) in the reference poloidal plane (left) and of the corresponding $\chi_{\lambda}$ (middle) and $\xi_{\lambda}$ (right) families in the image plane.

where $c$ is a dimensionless constant close to unity which controls the denoising sensitivity. At stage $n$ all the coefficients $s_{\xi_{\lambda}}$ whose absolute value is lower than $\Theta_{n}$ are discarded from Eq. 3.11 to calculate the next term of the sequence, that eventually converges to a limit. The $n+1$ term reads

$$
\Theta_{n+1}=\frac{c^{2}}{N_{n}} \sum_{\lambda /\left|s_{\xi \lambda}\right| \geq \Theta_{n}}^{N_{n}} s_{\xi_{\lambda}}^{2}
$$

where $N_{n}$ is the number of coefficients $s_{\xi_{\lambda}}$ larger or equal to $\Theta_{n}$. More details can be found in the Refs. [13, 19].

Last, the typical calculation time on a standard computer and without any optimisation of the algorithm for a $64 \times 64$ mesh grid in the reference poloidal plane is about 5 minutes for the calculation of the look-up table, two hours for the calculation of the transfer matrix $K$ and its inversion, and then five seconds per image (assuming that the magnetic field is constant over the extract of the movie processed) to obtain the reference poloidal plane after denoising, i.e. about fourteen hours for a 10000 image movie.

\section{Validation}

Sources of possible errors in such a tomographic reconstruction are numerous: errors in the code, mathematical typos, wrong assumptions (especially assuming a constant emissivity along the field lines), noise amplification, noninversability of the $K$ matrix. We propose in this section three different validation levels of the method. First, we will show that there are no mathematical or code errors in the program by reconstructing an academic case with additional noise to further check the thresholding method. Second, taking a more relevant configuration from a TOKAM3X simulation [20], we show that the reconstruction is valid even in the case of a slowly varying emissivity along the magnetic field lines. Third, the reconstruction is applied to real data obtained on the COMPASS tokamak and is compared to probe data to validate the method under experimental conditions.

\subsection{Academic case}

Let consider the simple case of a single node structure in the mesh grid of the reference poloidal plane, as shown in Fig. 3.1 (left). The synthetic image seen by the camera is given by the central picture in Fig. 3.1. To test the thresholding method proposed in Ref. [13], we add a strong white noise (gaussian noise having a standard deviation of $32 \%$ of the maximum pixel intensity of the total image) to the picture and obtain Fig. 4.1 (a). We then apply the tomographic reconstruction described in the previous section and present two images (Fig. 4.1 (c) and (d)). Fig. 4.1 (c) represents the coefficients $s_{\xi_{\lambda}}$ associated to each node of the poloidal plane (see Eq. 3.9) that will be called $S_{\text {threshold }}$ in the following sections. This image serves to understand how the thresholding method works and shows that, even without projecting on a wavelet basis, the coefficient corresponding to the relevant signal is well above the ones corresponding to the white noise. Applying the threshold procedure with the parameter $c$ being set to 3.5, the coefficients $s_{\lambda}$ (see Eq. 3.7 and 3.8) associated to each node of the poloidal plane are plotted in Fig. 4.1 (d), that we will be called $S_{\text {rec }}$ in the following. One can see that this image corresponds to the reconstructed structures in the reference plane taken at the beginning of the procedure (Fig. 3.1, left). The value of the single point node after tomographic reconstruction is equal to 1.3 whereas it was set to 1 initially, implying that the white noise added a $30 \%$ error on the intensity value reconstruction. In addition, the reconstructed image in Fig. 4.1 (b) obtained after applying $K$ to the reconstructed poloidal plane shows how similar the picture is to Fig. 3.1 (middle), except 
a)
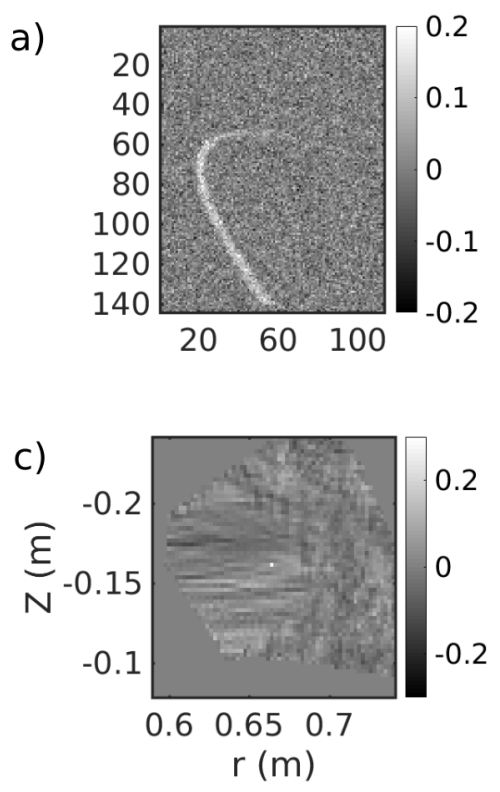

b)

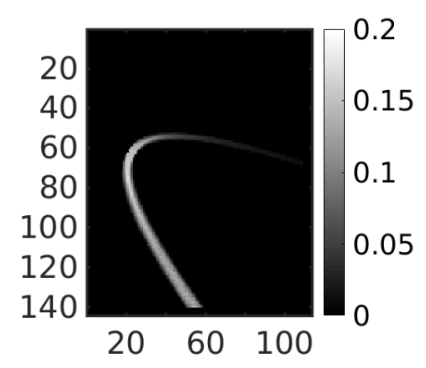

d)

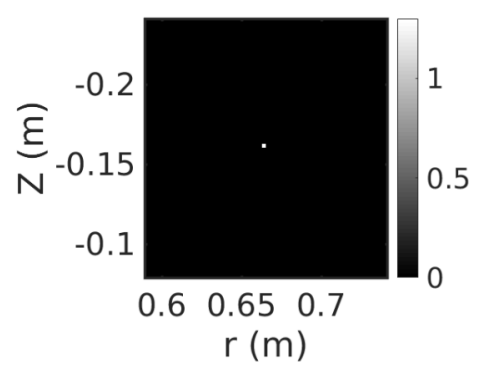

Figure 4.1: a) Noisy image generated after Fig. 3.1. b) Reconstructed image by applying $K S_{\text {rec }}$ c) Reconstructed poloidal plane $S_{\text {threshold }}$ and d) $S_{\text {rec }}$. Figure d) is obtained after thresholding $S_{\text {threshold }}$ with $c=3.5$.

from the slightly higher intensity level. This whole procedure validates the mathematical background and code and shows that even under strong noise corruption, the procedure can reveal the structure of the relevant signal.

In addition, more realistic structure shapes have been tested, namely circular and elliptical. In both cases, the reconstructed structures are slightly bigger than the original one, usually two pixels in radius larger. This is likely a consequence of the spatial gaussian filter applied, with a standard deviation of 5 pixels, to smear out the noise on the original camera image. As a consequence, it has been tested that two blob-like structures cannot be distinguished after reconstruction if they are not spatially separated by at least two pixels, independently on the shape or orientation of the structure. Last, it has been checked that there is no position shift of the reconstructed data compared to the synthetic ones in the region where most structures actually exists as obtained with experimental data (see Section 4.3).

\subsection{Reconstruction applied to TOKAM3X data}

We now take a case closer to experimental data by considering 3D data generated from the TOKAM3X code [20]. In particular, we consider a virtual camera able to record a signal proportional to the electron density generated by the code. It is interesting to point out that the density from the 3D data is not constant along the field lines within the field-of-view of the virtual camera and varies within the $20 \%$ range, so that the reconstruction will show how robust the method is in case of structures with an emissivity varying along the magnetic field lines. In Fig. 4.2 (b) and (c), we show the poloidal plane $S_{\text {ref }}$ of the density fluctuations taken as the reference plane and the corresponding image on the camera obtained by computing $I_{0}=K S_{\text {ref }}$, using the 3D data output from the code. Notice the high spatial resolution that the TOKAM3X code produces. We then apply the tomographic reconstruction to the image $I_{0}$ and obtain the reconstructed plane $S_{\text {rec }}\left(s_{\lambda}\right.$ coefficients from Eq. 3.8) in Fig. 4.2 (d) thresholded using $c=2$. For comparison, we show the interpolation of $S_{\text {ref }}$ over the same grid as $S_{\text {rec }}$ in Fig. 4.2 (e). In Fig. 4.2 (f), the reconstructed image $I_{\text {rec }}=K S_{\text {rec }}$ is shown for comparison with $I_{0}$. It can be stated that the reconstruction is able to recover the important features of the main poloidal plane. In addition, even though the resolution of reconstruction is lower (32x64) than the one from the TOKAM3X code, the two pictures in Fig. 4.2 (c) and (f) resembles well, showing that the most important parts of the signal have been extracted. This is further supported by the cross-correlation of these two pictures, shown in Fig. 4.2. The spatial maximum of correlation is high (almost 0.8) and is obtained for a zero shift in both the $\mathrm{X}$ and $\mathrm{Y}$ directions (red circle). The method thus seems reliable even in the more realistic case of structures extended along the field lines with slowly varying emissivity. 
a)

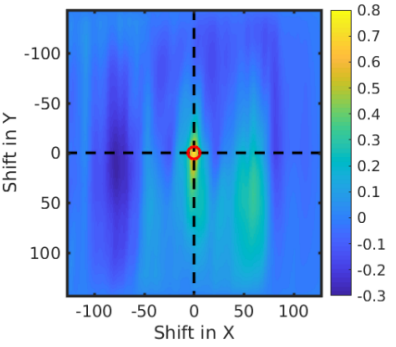

d)

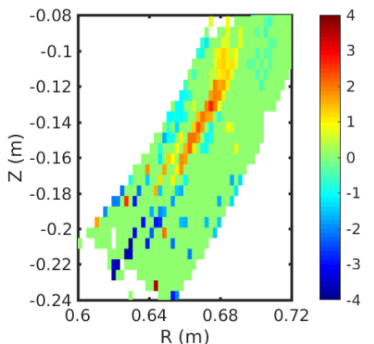

b)

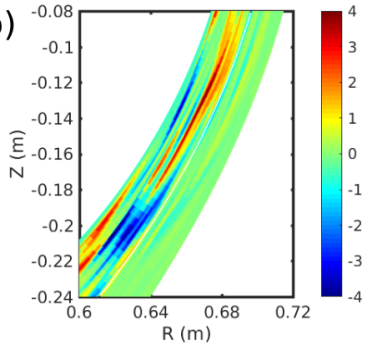

e)

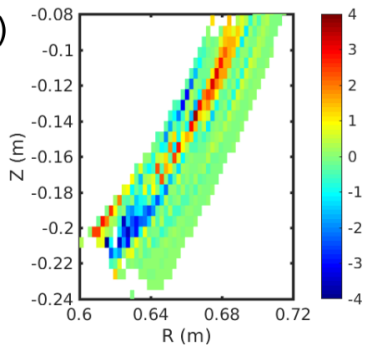

c)

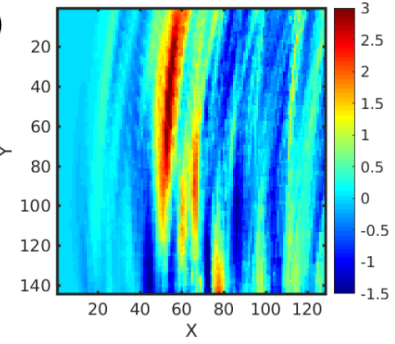

f)

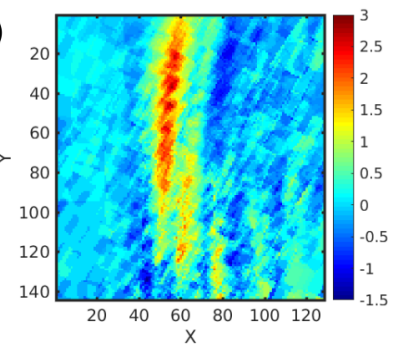

Figure 4.2: Tomographic reconstruction validation with TOKAM3X data. (a) Cross-correlation between the synthetic image (c) and the reconstructed image (f), the red circle shows the maximum of correlation, (b) Poloidal plane of reference $S_{\text {ref }}$ obtained from the TOKAM3X code, (c) synthetic image obtained after applying $I_{0}=K S_{\text {ref }},($ d) reconstructed poloidal plane $S_{\text {rec }}$ using $c=2$, (e) interpolation of $S_{\text {ref }}$ over the same grid as $S_{\text {rec }}$, (f) reconstructed image obtained by $I_{\text {rec }}=K S_{\text {rec }}$.

\subsection{Real data: validation with probes}

We now apply the reconstruction method to real data obtained in the COMPASS tokamak under the experimental configuration presented in the first section. The data were measured during the D-shape ohmic L-mode discharge \#15487 with a main electron density of $3-4 \times 10^{19} \mathrm{~m}^{-3}$, a toroidal magnetic field of $-1.15 \mathrm{~T}$ and a plasma current of $180 \mathrm{kA}$. In that case (negative magnetic field and positive plasma current) and in the LFS of the machine, the fieldlines are going down when rotating in the anticlockwise direction (for $\varphi$ increasing in Fig. 2.1). In order to compare the video data and probe data, we reconstructed 10000 frames that correspond to about $37 \mathrm{~ms}$. The time of the first image corresponds to $\sim 1137 \mathrm{~ms}$, selected in the middle of the flattop phase of the discharge (phase about $180 \mathrm{~ms}$ long). For each image of the 10000 frame video, a sliding median image calculated over 10 frames was subtracted and a Gaussian filter with a standard deviation of 5 pixels was applied, as already evoked in Section 2. In addition, camera pictures were shrunk to remove pixels for which no plasma was visible, either because the port edge, where the mirror is inserted, is in the way or either because the mirror was not big enough to cover the whole field of view of the camera. The poloidal plane that one is willing to reconstruct can be any plane of the torus (provided that the assumption of constant emissivity holds up to that plane). Here, we have chosen the plane at toroidal angle $\varphi=55$ degrees, i.e. the plane for which most of the LOS of the camera are tangential to the magnetic fieldlines. The reconstructed plane was discretized by a cartesian grid of $(R, Z)=(64,64)$ cells, giving a spatial resolution of $2.4 \mathrm{~mm}$ in the horizontal direction and $2.5 \mathrm{~mm}$ in the vertical one.

\section{Snapshot example}

In Fig. 4.3, we show a reconstruction example performed for the frame 54200 (time=1150.74 ms) of the selected time period. The camera data after the median and the Gaussian filtering are shown in Fig. 4.3 (a), while Fig. 4.3 (b) shows the reconstructed plane $S_{\text {threshold }}$ (coefficients $s_{\xi \lambda}$ in Eqs. 3.7 and 3.9) and Fig. 4.3 (c) shows the coefficients $s_{\lambda}$ after the thresholding method was applied with $c=3.5$ (the denoised reconstructed structure in the reference poloidal plane $S_{\text {rec }}$ ). In both figures, the red line corresponds to the last closed flux surface from the EFIT reconstruction software for that particular discharge time. The last image, Fig. 4.3 (d), shows the calculated image in the camera plane $I_{\text {rec }}=K S_{\text {rec }}$ obtained by applying the transfert matrix to the reconstructed structure $S_{\text {rec }}$. One can note that the reconstructed image $I_{\text {rec }}$ resembles to the original one, supported by the fact that the cross-correlation between these two images has a maximum above 0.8 and is not spatially shifted. In addition, on $I_{\text {rec }}$ a zero value rectangle is visible in the bottom left part of the image that corresponds to one edge 
a)

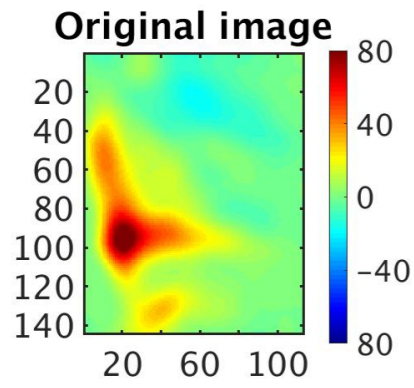

b) Threshold plane

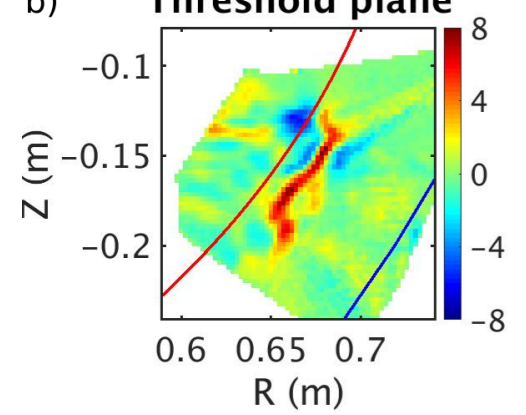

d)

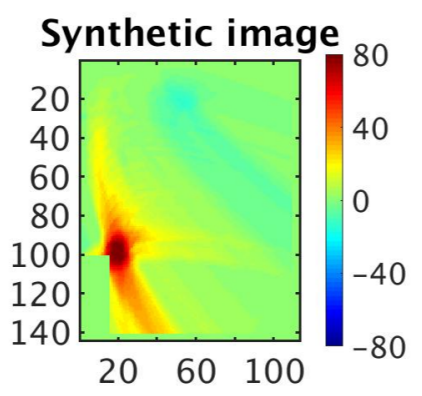

c)

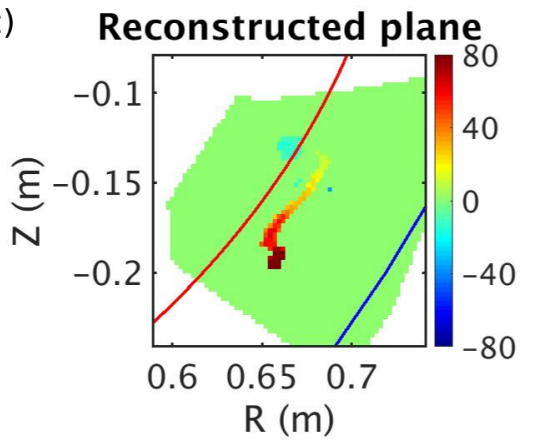

Figure 4.3: Tomographic reconstruction example for a snapshot taken at $1150.74 \mathrm{~ms}$ (frame 54200) of discharge \#15487. (a) Camera data subtracted from a sliding median taken over 10 frames around the considered image and for which a Gaussian filter with a standard deviation of 5 pixels was applied, (b) reconstructed poloidal plane $S_{\text {threshold }}$, (c) reconstructed poloidal plane $S_{\text {rec }}$ obtained applying the threshold method with $c=3.5,($ d) reconstructed camera image from $I_{\mathrm{rec}}=K S_{\mathrm{rec}}$. The red line in figures (b) and (c) is the position of last closed flux surface given by the EFIT reconstruction while the blue line is the vessel wall position.

of the mirror. Remember from Section 3.3 that the LOS collecting no light from the plasma should be discarded. A positive elongated structure is dominant on the reconstructed plane in Fig. 4.3 (c) but three negative structures $(1$ big and 2 small) are also visible, above the thresholding level and therefore relevant. It is also interesting to notice that the intensity of the elongated positive structure varies from its top edge to its bottom edge. This aspect will be commented in Section 5 .

\section{Validation with probes}

To further convince the reader that the tomographic reconstruction extracts the important part of the data on more than one snapshot, we have compared the reconstructed data with signals coming from Langmuir probes. The divertor of COMPASS was recently equipped [21] with a 54 Langmuir probe array measuring ion saturation currents (-270 V applied to the probes) at a sampling frequency of $4 \mathrm{MHz}$ and located at $\varphi=127.5$ degree from the reconstructed plane. At that location, mainly two probes are magnetically connected to the part of the reference poloidal plane visible in the camera field, so-called LPA46 and LPA47 in the COMPASS database. In Fig. 4.4 (left column), the maximum of correlation of the signal coming from these two probes and each pixel of the poloidal plane is presented. In addition, we show for comparison the correlation of the camera data with the probe LPA39 which is not magnetically connected to the visible part of the reference plane. In Fig. 4.4 (right column), the delay associated to the maximum of correlations higher than $\mathbf{0 . 1}$ is given in microseconds, where a negative time delay means that the structure first reaches the probe before the reconstructed poloidal plane. Note that the probe signals were interpolated (downsampled) to match the time window and step of the 10000 frames considered for the analysis (270 kfps). The magnetic fieldline connected to the probe is also represented as a dashed red line and its ending point in the reference plane $(\varphi=55$ degrees) is shown as a red circle.

First, it is very interesting to see that the spatial maximum of correlation for the probe LPA47 is close to 0.4. Note that such correlation values coming from direct light/probe comparison were already observed on the TJ-K stellarator (see Fig. 4 of Ref. [22] for instance) or on the Alcator C-Mod machine [23]. On linear devices, correlation values up to 0.5 can often be observed for direct comparisons [24, 25]. It has also been shown [26] that selecting a relevant part of both probe and camera signals, high correlation values up to 0.8 can be found. A similar value is found here if we keep the frequencies between 13 and $17 \mathrm{kHz}$ 
corresponding to a peak in both probe and camera spectra. The magnitude of these correlations are linked to the dependency of both signal with the electron density and temperature but modelling of the light dependency with plasma parameters is out of the scope of this article. The spatial maximum value for the probe LPA46 is lower (about 0.3) because the probe is magnetically connected to an area of the reconstructed plane for which less signal is present. The signal to noise ratio is thus lower. On the other hand, the LPA39 probe that is not on any fieldline passing by the visible part of the reference poloidal plane has a weaker maximum of correlation, less than 0.13. It is important to add that the correlation between two consecutive probes on the divertor is strong (for instance higher than 0.7 between probe LPA46 and LPA47) but also non negligible between more distant probes (for instance almost 0.25 between LPA39 and LPA47). This probably explains why, even though weak, the probe LPA39 does not have a zero correlation value.

Second, the spatial maximum of correlation for the LPA47 probe is close to the point magnetically connected to the probe and the region of maximum correlation is very similar in size and shape to the reconstructed structures (see Figs. 4.3 and 5.1). On the other hand, for the LPA46 probe, the maximum of correlation is located above the point magnetically connected to the probe. This is first due to the fact that most of the signal of the $2 \mathrm{D}$ plane comes from a region above the point magnetically connected to the probe. Second, the crosscorrelation between a node located in the region where most of the signal is present and the whole reconstructed plane shows a similar pattern to what can be seen in Fig. 4.4, meaning that coherent structures travel poloidally on the reconstructed plane and explaining the correlation with a region not magnetically connected to the probe.

Third, when looking at the delay figures, one sees that for LPA46 and LPA47, the region corresponding to non-negligible correlation values depicts a color gradient, meaning that the time delay is evolving gradually from top (lower delay) to bottom (higher delay), and that structures are moving from top to bottom. This main average movement is visible by eyes when looking at the filtered camera data and is thus consistent with qualitative observations. It is also important to note that the time delay at the points magnetically connected to both probes is zero, even though the two points are at different poloidal location. Since the two probes are magnetically separated by about the same distance to the reconstructed plane $(2.4 \mathrm{~m})$, it is logical that the time delay is the same and it further validates the tomography reconstruction as different points in the $2 \mathrm{D}$ plane correspond to their conjugate on the divertor target. As a consequence, we consider from this last experimental checking and the ones in the two previous sections that the reconstruction method is valid and reliable.

\section{Influence of the accuracy in the magnetic field reconstruction}

The tomographic method is based on the magnetic field reconstruction provided by the EFIT software that uses different experimental magnetic and current measurements. This reconstruction has a certain precision and we investigate here what can be the effect of the latter on the tomographic inversion. Mainly, we have looked at the influence of two different changes: what happens when the whole magnetic reconstruction is shifted by few centimeters in the radial direction and what happens when the pitch angle between the poloidal and toroidal magnetic field is changed.

A radial shift in the interval of the whole magnetic structure yields hardly any change in the tomographic inversion for the reference plane we used in that work. The effect of the pitch angle change (we considered a $10 \%$ increase) is more sensitive. It changes mainly the sizes and the orientation of the structures. This higher sensitivity to the pitch angle change is due to the fact that two close magnetic field lines are almost parallel. Hence replacing one of them by the other as a result of the translation of the whole magnetic structure (radial shift) does not change too much the shape of the filament, at least in the vicinity of the reference poloidal plane. However, a change in the pitch angle modifies the slope of the fieldline even near the reference poloidal plane. As reported in section 4.3, the reference poloidal plane used for this calculation is close to the plane for which most of the LOS are tangential to the magnetic field lines ( $\varphi=55$ degree).

We also tested the robustness of the tomographic inversion to magnetic reconstruction errors when another reference poloidal plane is used ( $\varphi=80$ degree). The sensitivity of the tomographic reconstruction to both types of change (radial translation and pitch angle change) is higher. This can be explained with the following argument. When a LOS is tangent to a magnetic fieldline, a long interval of this fieldline is collected by the same pixel, whereas the contribution of the other parts of this same fieldline will be spead over a large number of pixels. Thus, tangential LOS will collect more signals. If the reference poloidal plane is a plane where most of the LOS are tangential to the magnetic fieldlines, then, as the reconstructed fieldlines are hardly different from the true one in the vicinity of the reference plane at the precision of the mesh grid, all the pixels where the corresponding signal is strong will be properly affected. Pixels further away might be wrongly affected but, as they correspond to much weaker signal, do not change significantly the tomographic reconstruction. Conversly, if the reference poloidal plane is far from the plane for which most of the LOS are tangential to the magnetic fieldlines, the error on the fieldline reconstruction 

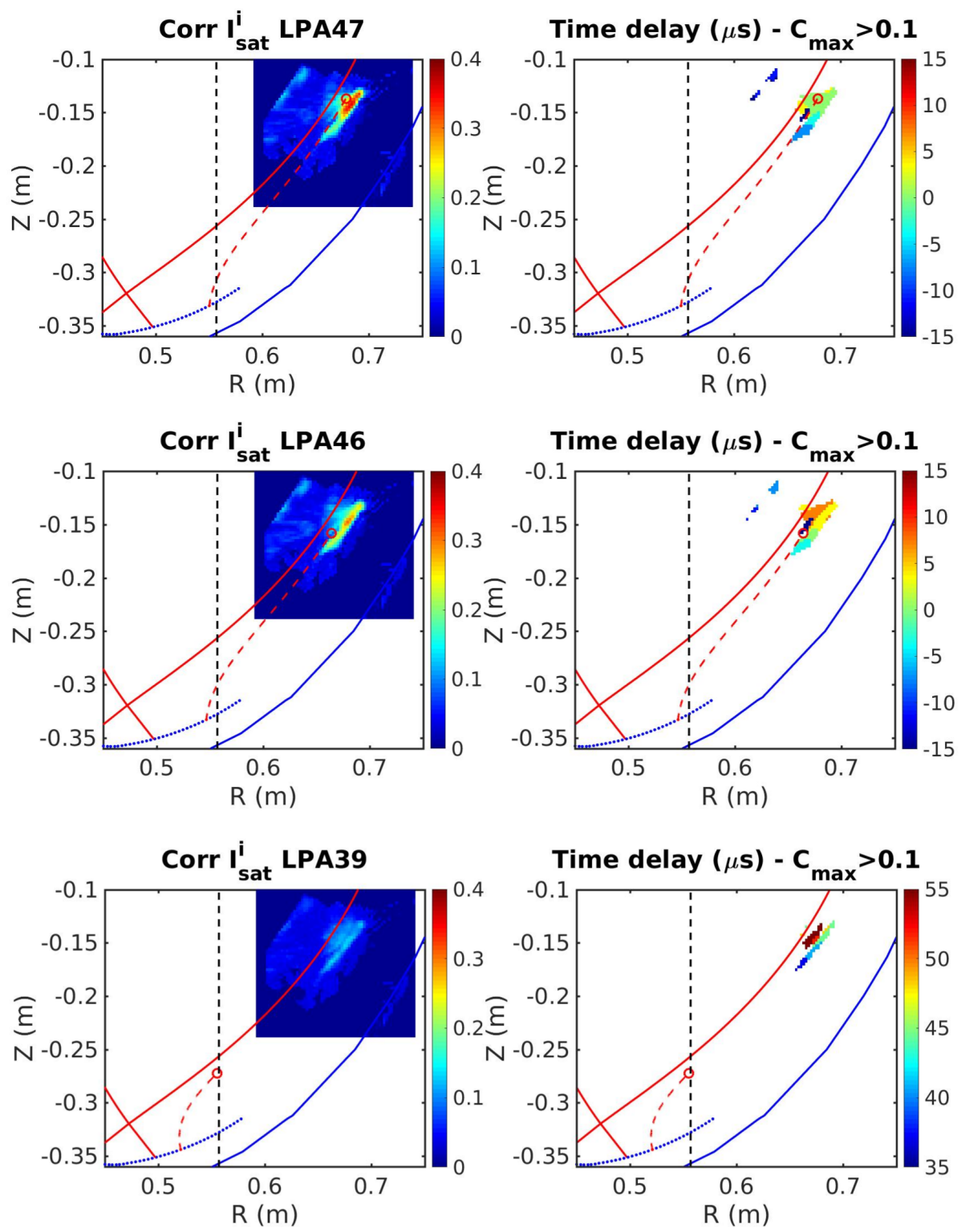

Figure 4.4: Maximum of correlation and associated delay between the ion saturation current measured by probes and the reconstructed camera data. Left column: normalized correlation value, right column: associated time delay in microseconds and for maximum of correlations higher than 0.1. Correlation for LPA47 (top), LPA46 (middle) and LPA39 (bottom). The red line represents the separatrix, the blue line, the vessel, and the dashed red line the magnetic fieldline starting from the probe position and finishing in the reconstructed plane (here $\varphi=55$ degree), as denoted by the red circle. 
will be larger than the mesh size in the plane that contributes the most to the signal and the reconstruction will be significantly distorted. As a consequence, even if theoretically any plane can be chosen as the reference poloidal plane for tomographic reconstruction, choosing a plane for which most of the LOS are tangential to the magnetic fieldlines makes the invertion more robust to magnetic field reconstruction errors.

\section{$5 \quad$ First investigation of the properties of the reconstructed fluctuations}

The tomographic reconstruction of plasma fluctuations from single camera data offers interesting perspectives. First, the visualization of the structures in a poloidal plane enables direct comparison with simulation data [20]. Second, a parallel objective is to ease the automatic processing of the videos in order to carry out the analysis of full length videos instead of focusing on some short sequences of interest. Ordinary, such sequences aim at illustrating rather basic aspects of a given phenomenon. As a result, one of the main criteria for selecting sequences of interest is the simplicity for the reader to understand the sequence which is depicted. It is however usually unclear whether the sequence is representative of the turbulence properties in the whole video and there is a significant risk to oversimplify the overall picture of the physics behind. It would be therefore of great interest to analyze complete shot videos under various discharge conditions in order to assess whether such sequences are statistically representative of the turbulence properties or not.

For such a reason, the TRACK software [27] has been chosen to analyze videos of reconstructed data. TRACK has evolved from the TRACE code already used in fusion research for investigations of plasma-wall interactions [28]. The detection is based on auto-adaptive thresholding techniques which enable an efficient automatic detection in various conditions met in our experiments, while the tracking is based on predictive Bayesian methods. The comparison of automatic analysis results with manual tracking of the plasma structures in 3000 frames shows only minor differences, concentrated on sequences where the coexistence of several structures does not give any certainty about the correct trajectories. The comparison of manual and automatic tracking for structures followed on 5 or more consecutive frames show no difference. In the following, we present some of the first results obtained with this approach, that shows the potential of the tomographic inversion coupled with a powerful detecting and tracking software.

\section{Illustrative sequence}

In Fig. 5.1, we present 10 successive reconstructed poloidal planes located at $\varphi=55$ degrees and measured during the discharge \#15487. From frame 54195 (time $\sim 1150.72 \mathrm{~ms}$ ) to frame $54204(\sim 1150.76 \mathrm{~ms}$ ), one can see the appearance of a positive structure that moves poloidally and radially, before getting elongated in the poloidal direction and disappearing at some radial position. Starting from frame 54200, a negative structure appears and experiences a similar movement. In particular, the radial position where the structures get elongated and disappear is almost the same for both structures. This is a first hint on where the shear flow layer could be for that particular shot, about $1.5 \mathrm{~cm}$ outside from the separatrix calculated by the EFIT code. Note that they are also evidences from mid-plane measurements with horizontal reciprocating probes that the shear flow region is located about 1 or $1.5 \mathrm{~cm}$ ahead of the separatrix position given by the EFIT code. Then, these structures are automatically tracked with TRACK over 5 frames $(18.5 \mu \mathrm{s})$. For the positive structure, the total displacement is about $3.9 \mathrm{~mm}$ in the radial direction and $22.6 \mathrm{~mm}$ in the poloidal direction, with a maximum radial velocity of $900 \mathrm{~m} / \mathrm{s}$ and a maximum poloidal velocity of nearly $3.5 \mathrm{~km} / \mathrm{s}$ in the last part of the trajectory. Note that we refer to radial velocities for velocity components locally perpendicular to the magnetic surfaces, while we refer to poloidal for velocities tangential to these surfaces.

We now come back to the observation we made about the elongated structure visible in the frame 54200 (see Fig. 4.3). It is clear from the previous and next frames that the structure experiences a very fast movement as it probably reaches a shear flow layer. This most likely explains why the structure in frame 54200 shows an intensity gradient: during the recording time of the camera, it moves very fast downwards tangentially and at some moment moves away perpendicularly. The whole movement happens in a very short time, probably shorter than the exposure time of the camera that is only $2.1 \mu \mathrm{s}$. It indicates that the camera was not yet recording fast enough to fully resolve the dynamic near the shear flow region and should be set at least twice faster. 

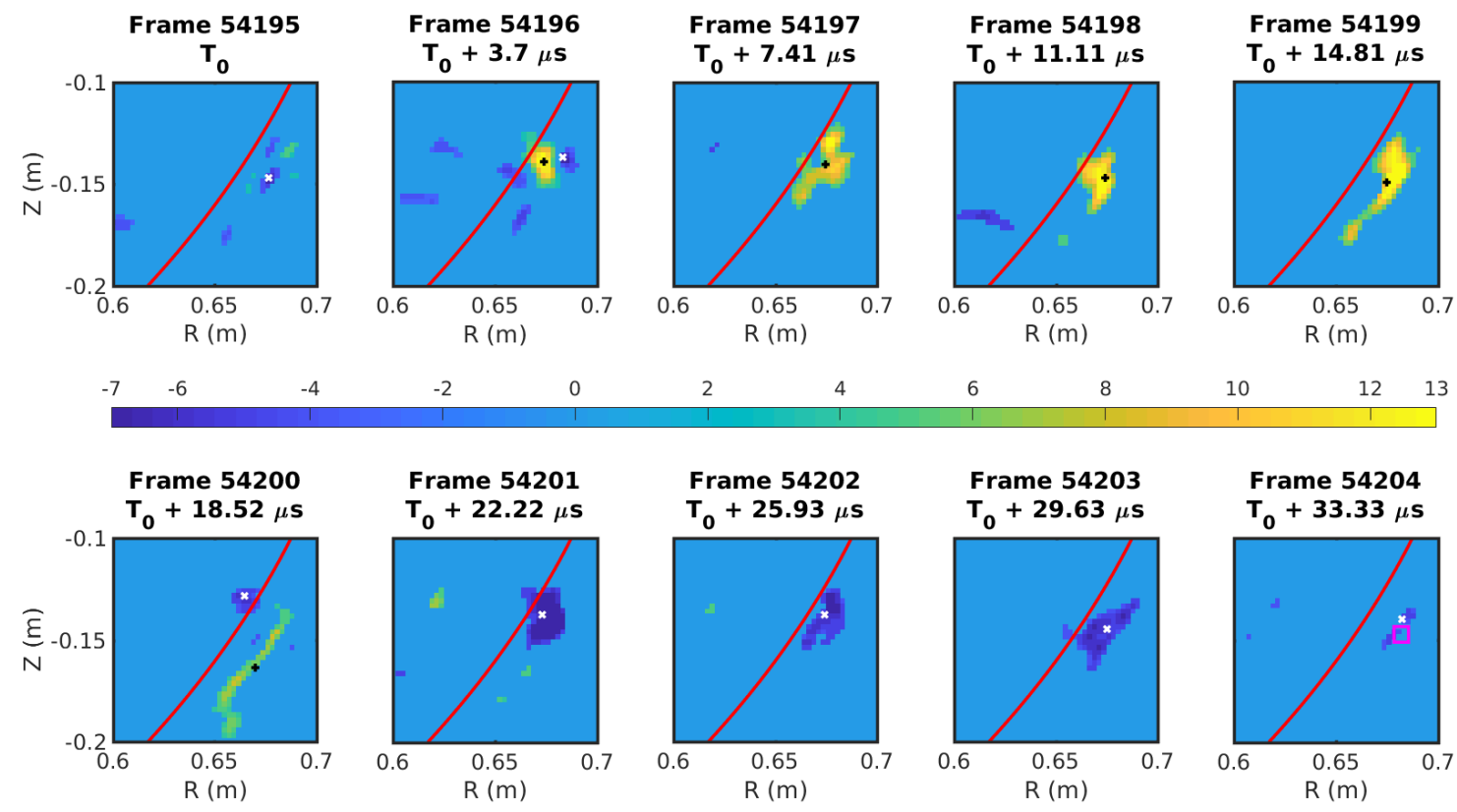

Figure 5.1: Reconstructed poloidal planes at $\varphi=55$ degrees for 10 frames taken from the discharge \#15487 around $T_{0} \sim 1150 \mathrm{~ms}$. The thresholding method was applied with $c=3.5$. The black $(+)$ and white $(\mathrm{x})$ crosses indicate the centre of mass of positive and negative structures, respectively, automatically detected and tracked by the TRACK software. The red line indicates the position of the separatrix from EFIT reconstruction. The pink rectangle corresponds to the zone chosen to obtain Fig. 5.2. 

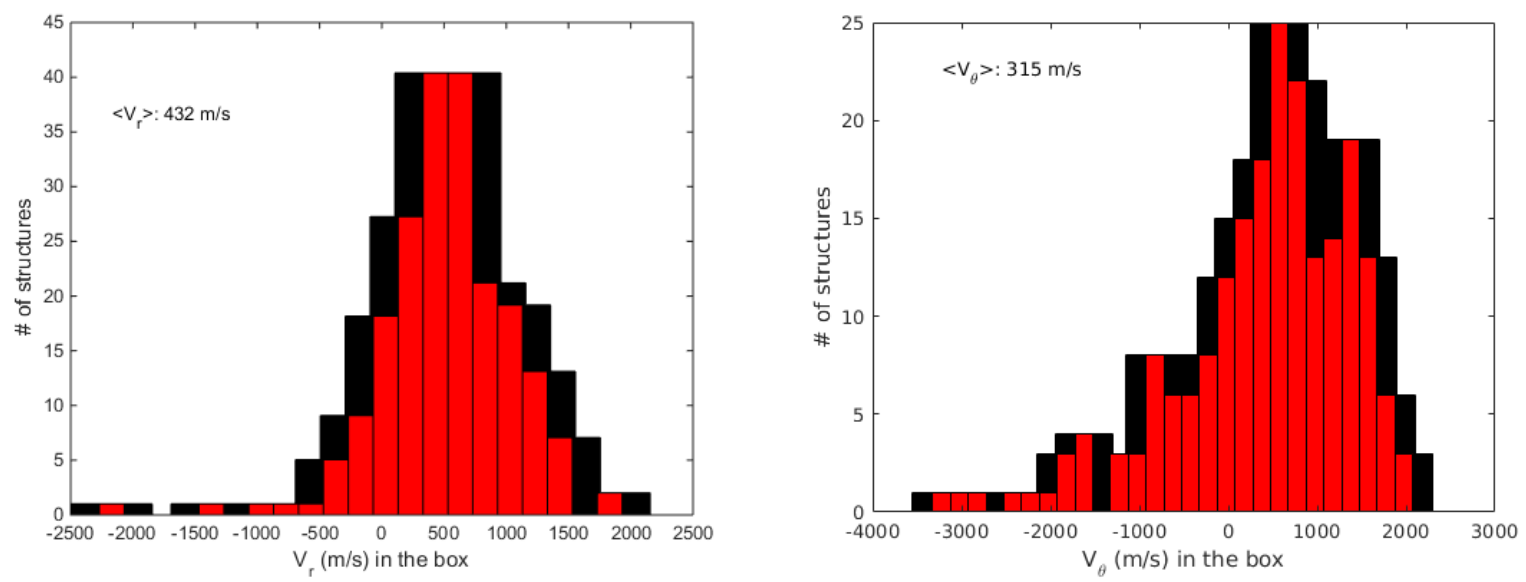

Figure 5.2: Radial and poloidal velocity distributions (left and right, respectively) calculated with TRACK in the small region delimited by the pink rectangle in figure 5.1. The step of the red color distribution is $200 \mathrm{~m} / \mathrm{s}$, while the black bars represent the broadening of the distribution assuming a systematic one pixel error detection of the structures $( \pm 650 \mathrm{~m} / \mathrm{s})$. The average radial and poloidal velocities are also indicated on the picture.

\section{Evidence for complex turbulent dynamic}

In order to go beyond the exploration of a short sequence, a full video composed of 10000 frames (37 ms) has been analyzed with the TRACK software (the same video sequence as used in Section 4.3). In total, 3637 positive structures and 3811 negative structures have been automatically detected and tracked and the code main ouputs are their velocity, size, orientation and aspect ratio. Here, we will only present an example of what the velocity distribution functions can be. A more sophisticated analysis will be presented in a separate paper.

For this analysis, we only consider the 626 positive structures that can be tracked on at least 5 consecutive frames. In Fig. 5.2, we present the radial and poloidal velocity distributions obtained for these structures and from a small rectangular region of $7.2 \times 7.6 \mathrm{~mm}^{2}$, highlighted by a pink rectangle in Fig. 5.1 (frame 54204). This region corresponds to the one where the correlation with the probe LPA47 depicts a maximum as presented in the top image of Fig. 4.4. Note that positive velocities $v_{r}$ and $v_{\theta}$ mean outwards and downwards, respectively.

As can be seen in Fig. 5.2, the distributions are quite wide with $v_{r}$ ranging from -500 to $1500 \mathrm{~m} / \mathrm{s}$ and $v_{\theta}$ from -2000 to $2000 \mathrm{~m} / \mathrm{s}$, values coherent to what can usually be observed on COMPASS [29, 30]. It is interesting to note that most structures are statistically experiencing a movement outwards and downwards but that some of them move in the opposite direction, mostly upwards but some of them also inwards, with non-negligible velocities. This behavior is not surprising in a turbulent media and was reported in many edge turbulence codes (see Ref. [31] for instance), but can only be investigated by individually tracking single structures movements. It appears that the turbulent structure depicted in Fig. 5.1 is amongst the $25 \%$ fastest structures in the perpendicular direction and the fastest structure in the tangential direction. Despite its qualitative behaviour seems representative of many other observed structures, it is certainly not the case from a quantitative point of view. In addition, the existence of counter-propagating structures at the same location demonstrates that the turbulence dynamics is actually more complex than what a short sequence suggests. Last but not least, it is important to point out that conditional averaging techniques usually applied to gas-puff imaging data [7] would show the main motion outwards and downwards with an average velocity of order of few hundreds meters per second, hiding the real structure motion.

\section{Conclusions and perspectives}

The tomographic reconstruction technique presented in Ref. [13] for circular plasmas was successfully applied to camera data recorded at a frame rate of $270 \mathrm{kfps}$ during a D-shaped plasma discharge of COMPASS. The robustness of the method was tested using slowly varying emissivity signals along the field lines (up to $20 \%$ on the total field of view of the camera) coming from a TOKAM3X simulation [20]. It shows that the main poloidal features can be well retrieved, as the cross-correlation between the input synthetic image and the one reconstructed shows a very high maximum of correlation and no spatial shift. Then, the inversion of real camera data was 
compared to the ion saturation currents measured by different probes in the divertor of the COMPASS tokamak. Probes that are magnetically connected to the reconstructed poloidal plane show a high normalized correlation, up to 0.4 , which is a strong value for two diagnostics measuring different physical quantites and magnetically separated by about $2.4 \mathrm{~m}$. On the other hand, probes that are not magnetically connected to the plane show a very low correlation. The method is further validated by looking at the time delay obtained from the maximum of correlation at the position of the poloidal plane magnetically connected to the divertor probes. Its values stay constant for different probes, even though the position magnetically linked to the probe in the poloidal plane changes and the distance along the field line stays constant. This confirms that the reconstruction method correctly redistributes the camera signal at the right location on the poloidal plane. Investigation of the spatial resolution using synthetic data show that the structure size after reconstruction is 2 pixels wider in radius than initially and that two structures cannot be distinguished if they are closer to each other than 2 pixels. This effect is likely a consequence of the spatial gaussian filter applied to smear out the noise of the camera pictures and contributes to the error bars of the method.

The reconstructed data can then be used to study edge tokamak physics with some advantages compared to what other diagnostics can offer, such as gas-puff imaging or probes. For instance, unlike gas-puff imaging [32] or probes [33,34, 35], the light observation coming from the interaction of the neutral gas naturally present in the SOL is completely passive and non-perturbative. In addition, the spatial resolution in the radial and poloidal directions can be much higher than what probes can provide. Moreover, this technique does not bound the observation in one given poloidal plane but any poloidal plane within the field of view of the camera can be reconstructed. Last, as shown in this article, the camera observes turbulent structures located near the separatrix, location where probes cannot measure for a long time without globally perturbing the plasma and being eroded. On the other hand, the method also has some disadvantages that one has to consider. The whole inversion method, letting appart for the code development, requires high computational resources, especially if the magnetic field is varying. In addition, as for GPI, no clear model exists to link the plasma edge emissivity to any physical quantity without knowing the electron density and temperature profiles, gas influx, impurity contents

etc... Furthermore, uncertainties in the reconstruction method linked to the difficult spatial calibration (errors of several millimeters) of the camera and uncertainties in the magnetic field reconstruction (error in position of about $1 \mathrm{~cm}$ ) can modify the dynamic of blobs in the reconstructed poloidal plane. In addition, even though improving fast, nowadays cameras are still on the technological edge to study fast movements that can happen at the shear flow location, as we have shown in Section 5. Last, the signal is localized only in a region where the interaction from the neutral gas and the plasma is strongest, limiting the region of interest. However, this region is localized near the separatrix which is a region of major interest.

An other problem to tackle is the automatic analysis of reconstructed video that often represents several gigabytes of data. Within this enormous amount of data, one has to automatically detect the turbulent structures and then track them. The contour of these structures is rather well defined, as one can see in Fig. 4.3 for instance, allowing to detect them easily. On the other hand, following the structure is more challenging as they change in shape, directions and intensity on frame to frame basis, especially near the shear flow region. The high acquisition speed of the camera helps to reduce this difficulty but improvement shall arise with new generations of fast and highly sensitive cameras. Nevertheless, it was shown that using the TRACK software [27], it is possible to efficiently analyze complete videos with fully automatic processing techniques. The automatic extraction of the main structures characteristics, such as their velocity, size, aspect ratio, and orientation, in a variety of discharge conditions offers many perspectives for a better understanding of turbulence in the vicinity of the separatrix. It can, for instance, provide statistically reliable experimental data enabling a more straightforward comparison with simulation results or theoretical models. In addition, comparison with other diagnostics can be performed and provide valuable insight. For instance, comparing statistical results obtained with this method and results obtained by standard gas-puff imaging diagnostic could help understanding the influence of neutrals on the edge turbulence. Last, MHD modes or instabilities influencing the blobs dynamic can also be investigated as instabilities can also be detected by visible light observations [36].

\section{Acknowledgment}

The first author would like to dedicate this work to Marguerite Hella (1922-2018). The authors would also like to thank the APREX team (Romain Baude and Mikael Desecures) for their strong support and help with the TRACK software [27]. The COMPASS tokamak operation was co-funded by the MEYS project no. LM2015045. This work has been carried out within the framework of the project COMPASS-U: Tokamak for cutting-edge fusion research (No. CZ.02.1.01/0.0/0.0/16_019/0000768) and co-funded from European structural and investment 
funds. Part of this work was performed using the HPC resources of GENCI-CINES (Grant A0030506912) and of the MARCONI-FUSION HPC (project HEAT). This work has also been carried out within the framework of the EUROfusion Consortium and has received funding from the Euratom research and training programme 2014-2018 under grant agreement No 633053 for the project WP17-ENR-CEA-08. The views and opinions expressed herein do not necessarily reflect those of the European Commission.

\section{References}

[1] S. J. Zweben, J. A. Boedo, O. Grulke, C. Hidalgo, B. LaBombard, R. J. Maqueda, P. Scarin, and J. L. Terry. Edge turbulence measurements in toroidal fusion devices. Plasma physics and controlled fusion, 49:S1-S23, 2007.

[2] N. Fedorczak, P. Manz, S. C. Thakur, M. Xu, G. R. Tynan, G. S. Xu, and S. C. Liu. On physical interpretation of two dimensional time-correlations regarding time delay velocities and eddy shaping. Physics of plasmas, 19:122302, 2012.

[3] F.D. Halpern, P. Ricci, B. Labit, I. Furno, S. Jolliet, J. Loizu, A. Mosetto, G. Arnoux, J.P. Gunn, J. Horacek, M. Kocan, B. LaBombard, C. Silva, and JET-EFDA Contributors. Theory-based scaling of the SOL width in circular limited tokamak plasmas. Nuclear Fusion, 53:122001 (5pp), 2013.

[4] S. J. Zweben, J. L. Terry, D. P. Stotler, and R. J. Maquedan. Invited review article: Gas puff imaging diagnostics of edge plasma turbulence in magnetic fusion devices. Review of Scientific Instruments, 88:041101, 2017.

[5] I. Shesterikov, Y. Xu, M. Berte, P. Dumortier, M. Van Schoor, M. Vergote, B. Schweer, and G. Van Oost. Development of the gas-puff imaging diagnostic in the TEXTOR tokamak. Review of Scientific Instruments, 84:053501, 2013.

[6] I. Shesterikov, Y. Xu, C. Hidalgo, M. Berte, P. Dumortier, M. Van Schoor, M. Vergote, G. Van Oost, and the TEXTOR Team. Direct evidence of eddy breaking and tilting by edge sheared flows observed in the TEXTOR tokamak. Nuclear Fusion, 52:042004 (5pp), 2012.

[7] G. Fuchert, G. Birkenmeier, D. Carralero, T. Lunt, P. Manz, H. W. Müller, B. Nold, M. Ramisch, V. Rohde, U. Stroth, and the ASDEX Upgrade Team. Blob properties in L- and H-mode from gas-puff imaging in ASDEX upgrade. Plasma Physics and Controlled Fusion, 56:125001 (9pp), 2014.

[8] P. Tamain, H. Bufferand, L. Carbajal, Y. Marandet, C. Baudoin, G. Ciraolo, C. Colin, R. Futtersack, D. Galassi, P. Ghendrih, N. Nace, F. Schwander, and E. Serre. Interplay between plasma turbulence and particle injection in 3D global simulations. Contribution to Plasma Physics, 56(6-8):569-574, 2016.

[9] P. Tamain, G. Bonhomme, F. Brochard, F. Clairet, C. Gil, J. Gunn, P. Hennequin, G. Hornung, J. L. Segui, L. Vermare, $\mathrm{Ph}$. Ghendrih, and Tore Supra Team. Interaction of plasma transport and turbulence on particle fuelling. Journal of nuclear materials, 438:S148-S154, 2013.

[10] D. Iraji, I. Furno, A. Fasoli, and C. Theiler. Imaging of turbulent structures and tomographic reconstruction of TORPEX plasma emissivity. Physics of Plasmas, 17:122304, 2010.

[11] A. Fasoli, A. Burckel, L. Federspiel, I. Furno, K. Gustafson, D. Iraji, B. Labit, J. Loizu, G. Plyushchev, P. Ricci, C. Theiler, A. Diallo, S. H. Mueller, M. Podesta, and F. Poli. Electrostatic instabilities, turbulence and fast ion interactions in the TORPEX device. Plasma Physics and Controlled Fusion, 52:124020, 2010.

[12] M. Odstrcil, J. Mlynar, V. Weinzettl, P. Hacek, T. Odstrcil, G. Verdoolaege, M. Berta, T. Szabolics, , and A. Bencze. Plasma tomographic reconstruction from tangentially viewing camera with background subtraction. Review of Scientific Instruments, 85:013509, 2014.

[13] R. Nguyen Van Yen, N. Fedorczak, F. Brochard, G. Bonhomme, K. Schneider, M. Farge, and P. Monier-Garbet. Tomographic reconstruction of tokamak plasma light emission from single image using wavelet-vaguelette decomposition. Nuclear Fusion, 52:013005 (11p), 2012. 
[14] R. Panek, J. Adamek, M. Aftanas, P. Bilkoval, P. Bohm, F. Brochard, P. Cahyna, J. Cavalier, R. Dejarnac, M. Dimitrova, O. Grover, J. Harrison, P. Hacek, J. Havlicek, A. Havranek, J. Horacek, M. Hron, M. Imrisek, F. Janky, A. Kirk, M. Komm, K. Kovarik, J. Krbec, L. Kripner, T. Markovic, K. Mitosinkova, J. Mlynar, D. Naydenkova, M. Peterka, J. Seidl, J Stockel, E. Stefanikova amd M. Tomes, J. Urban, P. Vondracek, M. Varavin, J. Varju, V. Weinzettl, J. Zajac, and the COMPASS team. Status of the COMPASS tokamak and characterization of the first H-mode. Plasma Physics Control. Fusion, 58:014015 (9pp), 2016.

[15] A. Havranek, V. Weinzettl, D. Fridrich, J. Cavalier, J. Urban, and M. Komm. Implementation of rapid imaging system on the compass tokamak. Fusion Engineering and Design, 123:857-860, 2017.

[16] S. Silburn and et al. Calcam. Zenodo. http://doi.org/10.5281/zenodo.1478554, November 2018.

[17] P. Tamain, Ph. Ghendrih, H. Bufferand, G. Ciraolo, C. Colin, N. Fedorczak, N. Nace, F. Schwander, and E. Serre. Multi-scale self-organisation of edge plasma turbulent transport in 3D global simulations. Plasma physics and controlled fusion, 57(5):054014 (10pp), 2015.

[18] L. C. Appel, M. K. Bevir, and M. J. Walsh. Equilibrium reconstruction in the START tokamak. Nuclear Fusion, 41(2):169 (12pp), 2001.

[19] A. Azzalini, M. Farge, and K. Schneider. Nonlinear wavelet thresholding: a recursive method to determine the optimal denoising threshold. Applied and Computational Harmonic Analysis, 18:177-185, 2004.

[20] P. Tamain, H. Bufferand, G. Ciraolo, C. Colin, D. Galassi, Ph. Ghendrih, F. Schwander, and E. Serre. The TOKAM3X code for edge turbulence fluid simulations of tokamak plasmas in versatile magnetic geometries. Journal of Computational Physics, 321:606-623, 2016.

[21] J. Adamek, J. Seidl, J. Horacek, M. Komm, T. Eich, R. Panek, J. Cavalier, A. Devitre, M. Peterka, P. Vondracek, J. Stockel, D. Sestak, O. Grover, P. Bilkova, P. Bohm, J. Varju, A. Havranek, V. Weinzettl, J. Lovell, M. Dimitrova, K. Mitosinkova, R. Dejarnac, M. Hron, The COMPASS Team, and The EUROfusion MST1 Team. Electron temperature and heat load measurements in the COMPASS divertor using the new system of probes. Nuclear Fusion, 57:116017 (7pp), 2017.

[22] G. Fuchert, G. Birkenmeier, M. Ramisch, and U. Stroth. Characterization of the blob generation region and blobby transport in a stellarator. Plasma Physics and Controlled Fusion, 58:054005 (10pp), 2016.

[23] O. Grulke, J. L. Terry, B. LaBombard, and S. J. Zweben. Radially propagating fluctuation structures in the scrape-off layer of Alcator C-Mod. Physics of Plasmas, 13:012306, 2006.

[24] S. Oldenburger, C. Brandt, F. Brochard, N. Lemoine, and G. Bonhomme. Spectroscopic interpretation and velocimetry analysis of fluctuations in a cylindrical plasma recorded by a fast camera. Review of Scientific Instruments, 81:063505, 2010.

[25] G. Y. Antar, J. H. Yu, and G. Tynan. The origin of convective structures in the scrape-off layer of linear magnetic fusion devices investigated by fast imaging. Physics of Plasmas, 14:022301, 2007.

[26] O. Grulke, J. Terry, I. Cziegler, B. LaBombard, and O. Garcia. Experimental investigation of the parallel structure of fluctuations in the scrape-off layer of Alcator C-Mod. Nuclear Fusion, 54:043012 (12pp), 2014.

[27] R. Baude and M. Desecures. Track software. http://www.aprex-solutions.com/, 2018.

[28] F. Brochard, A. Shalpegin, S. Bardin, T. Lunt, V. Rohde, J.L. Briancon, G. Pautasso, C. Vorpahl, R. Neu, and The ASDEX Upgrade Team. Video analysis of dust events in full-tungsten ASDEX Upgrade. Nuclear Fusion, 57(3):036002 (13pp), 2017.

[29] O. Grover, J. Seidl, D. Refy, J. Adamek, P. Vondracek, M. Tomes, P. Junek, P. Hacek, J. Krbec, V. Weinzettl, M. Hron, S. Zoletnik, and the COMPASS team. Limit cycle oscillations measurements with langmuir and ball-pen probes on COMPASS. Nuclear Fusion, 58:112010 (12pp), 2018.

[30] J. Seidl, J. Krbec, M. Hron, J. Adamek, C. Hidalgo, T. Markovic, A.V. Melnikov, J. Stöckel, V. Weinzettl, M. Aftanas, P. Bilkova, O. Bogar, P. Bohm, L.G. Eliseev, P. Hacek, J. Havlicek, J. Horacek, M. Imrisek, K. Kovarik, K. Mitosinkova, R. Panek, M. Tomes, and P. Vondracek. Electromagnetic characteristics of geodesic acoustic mode in the COMPASS tokamak. Nuclear Fusion, 57:126048 (15 pp), 2017. 
[31] Ph. Ghendrih, Y. Sarazin, G. Attuel, S. Benkadda, P. Beyer, G. Falchetto, C. Figarella, X. Garbet, V. Grandgirard, and M. Ottaviani. Theoretical analysis of the influence of external biasing on long range turbulent transport in the scrape-off layer. Nuclear Fusion, 43:1013 - 1022, 2003.

[32] S. J. Zweben and S. S. Medley. Visible imaging of edge fluctuations in the TFTR tokamak. Physics of Fluids B: Plasma Physics, 1:2058, 1989.

[33] M.V. Umansky, D. Brunner, B. LaBombard, and T. D. Rognlien. Modeling of local edge plasma perturbations induced by a biased probe. Contribution to Plasma Physics, 52(5-6):417-423, 2012.

[34] C. Colin, P. Tamain, P. Ghendrih, F. Schwander, and E. Serre. Impact of a langmuir probe on turbulence measurements in the scrape-off-layer of tokamaks. Contribution to Plasma Physics, 54(4-6):543-548, 2014.

[35] D. Brunner, M.V. Umansky, B. LaBombard, and T.D. Rognlien. Divertor death-ray explained: An artifact of a langmuir probe operating at negative bias in a high-recycling divertor. Journal of Nuclear Materials, 438:1196-1199, 2013.

[36] Tomas Jirman, Matej Peterka, Jakub Seidl, Jordan Cavalier, Vladimir Weinzettl, Frederic Brochard, Pavel Cahyna, James Harrison, Martin Imrisek, Andrew Kirk, Radomir Panek, and the EUROfusion MST1 Team. Edge plasma study using a fast visible light camera in the COMPASS tokamak. Energy Procedia, 127:360-368, 2017. 